\title{
Effectiveness of Reverse Kangaroo Mother Care Position in Decreasing Adverse Physiological Events and Pain during Screening of Premature Infants for Retinopathy of Prematurity
}

Ma. Socorro Fatima P. Calitis, MD, ${ }^{1}$ Maria Esterlita T. Villanueva-Uy, MD, MSPH, ${ }^{2}$ Socorro De Leon-Mendoza, MD, ${ }^{3}$ Maria Angelica Villano, $\mathrm{MD}^{4}$ and Jazel Eleazer-Verde, $\mathrm{MD}^{4}$

\author{
${ }^{1}$ Section of Newborn Medicine, Department of Pediatrics, Philippine General Hospital, University of the Philippines Manila \\ ${ }^{2}$ Institute of Child Health and Human Development, National Institutes of Health, University of the Philippines Manila \\ ${ }^{3}$ Kangaroo Mother Care Foundation Philippines, Inc. \\ ${ }^{4}$ Department of Ophthalmology and Visual Sciences, Philippine General Hospital, University of the Philippines Manila
}

\begin{abstract}
Background. Retinopathy of prematurity (ROP) screening is uncomfortable and even painful in preterm infants.

Objective. To determine the effectiveness of the Reverse-Kangaroo Mother Care (R-KMC) position compared with the conventional position in decreasing adverse physiological events and pain during ROP screening.

Methods. This was a parallel randomized controlled trial with two arms- R-KMC position and conventional position (control). The primary outcomes included increased oxygen saturation, decreased heart and respiratory rates, and decreased pain intensity measured by Prematurity Infant Pain Profile (PIPP).
\end{abstract}

Results. There were 100 preterm infants recruited. There were no differences in baseline characteristics, mean ROP screening duration, mean physiological parameters after the mydriatic application and during ROP screening

Paper presented and won $2^{\text {nd }}$ place in the $2^{\text {nd }}$ International Symposium for Asia: The Neonate 2018, on Novermber 2018, at Shanghai, China.

Paper presented and won $2^{\text {nd }}$ place in the International Conference and $13^{\text {th }}$ Annual Meeting, Philippine Society of Newborn in Medicine, on February 6, 2019, at Manila, Philippines.

Paper presented and won $2^{\text {nd }}$ place in the University of the Philippines - Philippine General Hospital Interhospital Research Contest on October 2018, at Manila, Philippines.

Paper presented and won $2^{\text {nd }}$ place in the University of the Philippines - Philippine General Hospital Department of Pediatrics Reseach Contest on October 2018, at Manila Philippines.

Paper presented in the Congress of the Federation of Asia and Oceania Perinatal Societies - Integration of Best Practices in Perinatal Care on September 2018, at Manila, Philippines.

Corresponding author: Ma. Socorro Fatima P. Calitis, MD Section of Newborn Medicine

Department of Pediatrics

Philippine General Hospital

University of the Philippines Manila

Taft Avenue, Ermita, Manila 1000, Philippines

Email: soc_calitismd@yahoo.com between the two. Only the mean respiratory rates were significantly lower among the R-KMC group during the application of mydriatric agent. (50.26 \pm 6.18 vs. 51.6 \pm 8.35; $p=0.0191$ ). Relative risk (RR) in the control group is 37 to 84 versus 40 to 68 in the R-KMC group. The R-KMC group had a significantly lower mean PIPP score (6.5 \pm 2.06 vs. $8.8 \pm 2.80 ; p<0.0001)$ after mydriatic application and during ROP screening (7.6 \pm 2.05 vs. 9.8 $\pm 2.90 ; p<0.0001)$ compared with control. No apnea was observed after mydriatic application, but one apneic episode was in control during ROP screening. $26 \%$ of the ROP screeners recommended the R-KMC position during ROP screening, while a majority $(70 \%)$ was neutral.

Conclusion. R-KMC position, a low-cost intervention, significantly reduces pain during ROP screening. It is recommended as a position of choice during ROP screening.

Key Words: Reverse Kangaroo Mother Care position, Kangaroo Mother Care, ROP Screening, Premature Infant Pain Profile 


\section{INTRODUCTION}

\section{Background}

Neonates, especially preterm infants, are more sensitive to pain and are vulnerable to its long-term effects compared with older children and adults. Preterm infants display a lower threshold and more pronounced reflex responses to touch due to their immature peripheral and central nervous systems. ${ }^{1}$

Varying degrees of neonatal discomfort, stress, or pain may be associated with routine patient care. Routine eye examination to screen for retinopathy of prematurity (ROP) is a standard of care for preterm infants who are $\leq 32$ weeks gestation at birth or older preterm infants with an unstable clinical course. It is performed for early detection and treatment of advanced stages of ROP to prevent retina detachment and resulting blindness. The International Evidence-Based Group for Neonatal Pain included ROP screening as one of the painful procedures performed in the NICU. Furthermore, applying mydriatic eye drops to dilate the pupils before an ROP eye examination also has adverse effects. $^{2}$

Kangaroo Mother Care (KMC) is a non-conventional, low-cost method for newborn care based upon intimate skinto-skin contact between mother and baby. It has beneficial effects on neurological, autonomic, somatic, behavioral, and motor development. ${ }^{3}$ In addition, the infant's vital signs illustrate measurable changes upon initiation of $\mathrm{KMC}$. These include positive changes in sleep cycles, heart rate, feeding efficiency, respiratory rate, crying, and bonding between caretaker and infant.

\section{KMC on Physiological Events}

In a study by Bera et al., 265 mother-baby pairs were analyzed to determine the effect of KMC on vital physiological parameters of the low-birth-weight newborn. The mean temperature rose by about $0.4^{\circ} \mathrm{C}$, respiratory rate by 3 per minute, heart rate by $5 \mathrm{bpm}$, and oxygen saturation $\left(\mathrm{SpO}_{2}\right)$ by $5 \%$ following $\mathrm{KMC}$ sessions during the three-day observation. Moreover, abnormalities such as hypothermia, bradycardia, tachycardia, and low $\mathrm{SpO}_{2}$ were often corrected during the KMC sessions. The neonates showed more regular respiration and often fell asleep while in the $\mathrm{KMC}$ position. No neonate developed apnea during KMC. ${ }^{4}$

A clinical trial in India conducted by Deghani et al. on 53 neonates admitted in the NICU, KMC, compared with conventional position, showed that the average temperature variations and the arterial oxygen saturation rate between the two groups were significantly better the KMC group during the three-day observation. ${ }^{5}$ In contrast to Bera's results, there were no significant differences in the mean heart and respiration rate between the two groups:

Another study conducted by Sutar et al. revealed that infants had fewer bradycardia events per hour while being held in $\mathrm{KMC}$ position than infants under the radiant warmer
( $p=0.0153$ ). The infants on KMC also had significantly fewer apneic attacks and episodes of oxygen desaturations while being held than under the warmer $(p<0.001)$. They also had significantly fewer desaturation events or apnea episodes compared with control $(\mathrm{p}=0.0032){ }^{6}$

In a pilot study by Padhi et al. al, 20 preterm infants at risk for ROP were screened in Reverse Kangaroo Mother Care position, and there was at least a $10 /$ min change (either rise or fall) in the heart rate of 12 infants and the respiratory rate of 10 infants change during the ROP procedure. Five preterm infants had a reduction in blood oxygen concentration below $92 \%$. The changes in the heart rate and $\mathrm{SpO}_{2}$ were not statistically significant. However, the change in the respiratory rate was statistically significant. ${ }^{7}$

\section{KMC on Response to Pain}

In the same study of Padhi on ROP examination in R$\mathrm{KMC}$ position, 8 out of the 20 preterm infants being screened were assessed as being completely relaxed as evidenced by no eye squeezing and crying during the ROP screening, while ten were partially relaxed. Only two were assessed as not in the relaxed state. Chidambaram et al. studied the effect of KMC in reducing pain due to heel prick among preterm neonates using Prematurity Infant Pain Profile as an assessment tool. There were 50 neonates in the KMC group and 50 neonates in the control group. The PIPP scores at 15 and 30 minutes and the mean PIPP difference at baseline and 30 minutes were significantly less in the KMC group than the control group. ${ }^{8}$

Similarly, Johnston conducted a trial on 64 extremely preterm (28-31 6/7 weeks age of gestation) and 74 moderately preterm neonates (32-36 wks. AOG) on the effect of $\mathrm{KMC}$ on pain during a heel-lancing procedure. The preterm infants in the treatment group were held in KMC position for 30 minutes before and during the heel-lancing procedure. The preterm infants in the control group were placed inside the incubator in the prone position. PIPP scores across the first 90 seconds from the heel-lancing procedure were significantly lower by 2 points in the $\mathrm{KMC}$ condition in both the extremely and moderately preterm infants. PIPP scores 90 seconds after the lancing procedure were also significantly lower in the KMC group (8.871 vs.10.677; p<.001). There was a trend of lower PIPP scores at three different time points (30, 60, and 90 secs after the procedure) among the infants on the KMC position than the controls, but these did not reach statistical significance. ${ }^{9,10}$

In a study by Gao of 80 preterm infants less than 37 weeks AOG in North-East China, the effect of repeated Kangaroo Mother Care on a series of painful procedures such as heel sticks was assessed from blood collection to recovery phase. The heart rate was significantly lower, and the duration of crying and facial grimacing were both significantly shorter in the KMC group $(n=38)$ than the incubator group $(n=37) .{ }^{11}$

The effect of skin-to-skin contact among healthy fullterm infants during a Hepatitis $B$ vaccine injection was 
studied by Kotsandy. Similar to studies seen among preterm infants, the term infants reached a calmer behavioral state sooner and had a more rapid HR decrease when placed in the skin-to-skin contact during the injection. ${ }^{12}$

\section{KMC on Evaluator's Satisfaction}

Henreics-Munoz interviewed mothers and neonatal nurse providers on their perspective on the value of $\mathrm{KMC}$ and maternal-care partnerships on infant and maternal wellbeing. Sixty-three percent of the mothers, compared with only $18 \%$ of nurses, believed that KMC should be provided daily. Also, $90 \%$ of mothers, compared with $40 \%$ of nurses, firmly believed that mothers should be care partners. ${ }^{13}$

\section{Significance of the Study}

Numerous benefits of the use of KMC have been reported to be related to more stable physiological (cardiorespiratory stability) and behavioral (sleep) states and relief of procedural pain. Despite this evidence, the adoption of KMC as a routine clinical practice remains variable and underutilized. This study aims to adopt Kangaroo Mother Care during Retinopathy of Prematurity screening in preterm infants. However, a slight modification to the traditional $\mathrm{KMC}$ position (chest to chest) was made by placing the infant's back to the mother's chest (reverse KMC position: $\mathrm{R}-\mathrm{KMC}$ ) to facilitate ROP screening in this study.

\section{GENERAL OBJECTIVE}

To determine Reverse Kangaroo Mother Care's effect in decreasing adverse physiological events and pain during ROP Screening among preterm infants.

\section{Definition of Terms}

1. Reverse-KMC position - preterm infant's back on the mother's chest with the latter wearing a tube blouse to stabilize the position

2. Conventional (ROP) position - preterm infant supine placed on a flat surface

3. Adverse physiologic events - physiologic changes such as an increase in the heart rate, increase in the respiratory rate, or decrease in oxygen saturations

\section{METHODS}

\section{Study Design}

This study was a parallel randomized controlled trial with two treatment arms - reverse KMC position and conventional ROP position, conducted from January to September 2017. The Research Ethics Board panel approved the study protocol before the start of the study. Strict confidentiality was imposed, and the anonymity of participants was protected. Patients were number-coded. Only codes were used in data forms and databases. Data forms were stored in a secured, locked cabinet with access only to the primary investigator having access to these. Encoded data stored in computers were password protected.

\section{Recruitment}

After informed consent was obtained from the parents, all preterm infants weighing $<2500$ grams, on whom ROP screening was ordered in the chart, were recruited in the study. Recruitment of eligible participants was done by either the primary investigator or research assistant. All clinical management remained the same between the two groups except for the position for ROP screening.

Infants were excluded from the study if they were on any respiratory support, born with significant congenital disabilities, inotropic support, or nebulization.

\section{Sample Size}

Borm et al. suggested a sample size computation for ANCOVA wherein a design factor $\left(1-\rho^{2}\right)$ was introduced. ${ }^{14}$ There was limited information on the correlation $\rho$ of $\mathrm{KMC}$ and conventional position; therefore, the minimum possible value of $\rho$ was used. At $90 \%$ power and $5 \%$ level of significance, overall sample sizes computed were 58, 94, and 11 for respiratory rate, heart rate, and oxygen saturation, respectively, with values obtained from Bera's results of paired differences comparing physiological events of infants under KMC and conventional position on Day 1. Using Johnston's results comparing 90 seconds post lance PIPP scores of neonates under $\mathrm{KMC}$ (8.871) and conventional position (10.677), the sample size was 13 for each group using the PASS 2008 software with 1000 simulations. The maximum sample size computed was 94 , and to account for possible attrition of $5 \%$ of the sample, the final sample size needed was 99 . Final sample size decided was 100 (R-KMC: $n=50$ and conventional position: $n=50$ ).

The participants were randomly assigned to either reverse $\mathrm{KMC}$ position or conventional position. Assignments were placed in opaque brown envelopes and picked by another person not directly involved in the study.

\section{Study Arms}

The conventional position entailed the placement of the preterm on a flat surface where the ROP screening was done. The infant was swaddled and placed on the bassinet, as seen in Figure 1.

In Reverse-KMC, mothers wearing the $\mathrm{KMC}$ tube blouse were asked to lie supine on a flatbed. Infants wore a cap and diapers. The infants were placed between the mother's breasts in a supine position with the infant's back in skinto-skin contact with the mother's chest. The mothers were advised to keep their hands over their infant's arms and legs, as seen in Figure 2. This restrained the baby adequately with eyes facing straight ahead towards the examiner. Mothers assigned to the KMC group but refused to undergo the $\mathrm{R}-\mathrm{KMC}$ position were not included in the study. 


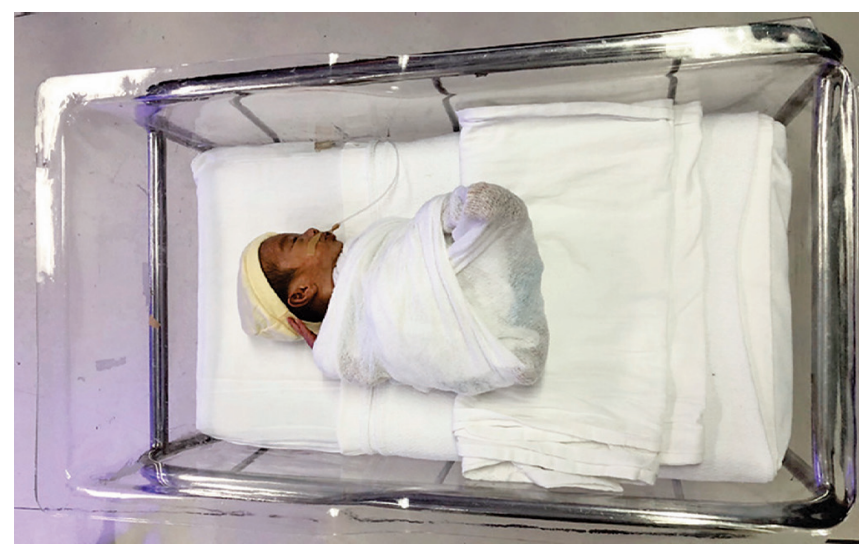

Figure 1. Conventional position.

\section{Pain Assessment}

Only two trained individuals performed pain assessment using the Prematurity Infant Pain Profile for the entire trial duration. PIPP is a validated neonatal pain assessment tool that included seven indicators in calculating a composite pain measure: 3 behavioral indicators (facial actions of brow bulge, eye squeeze, and nasolabial furrow), two physiological indicators (heart rate and oxygen saturation), and two contextual indicators (gestational age and behavioral state) to account for the changes in age over time. The PIPP scores ranged from 0-21 points. Scores $\leq 6$ indicated absence of pain or minimal pain; scores from 7-12: slight to moderate pain and scores > 12: severe pain.

Before the actual conduct of the trial, the two pain assessors were trained to ensure a high level of agreement between the two. The training involved pain assessment on 20 neonates. The assessors were in very strong agreement when a Cohen's kappa value greater than 0.80 was achieved.

\section{Physiologic parameters}

Before group assignment, physiological parameters such as oxygen saturation, heart rate, and respiratory rate using a cardiac monitor were recorded. A probe was placed on the right palm of the infant to monitor for oxygen saturation.

\section{ROP screening procedure}

The infant did not receive any feeding 1 hour before the examination. Pupillary dilatation was performed 45-60 minutes before screening by an Ophthalmology resident in both groups. One drop of the combination of phenylephrine $2.5 \%$ and tropicamide $0.5-1 \%$ was instilled in each eye. This was repeated every 5 minutes 2-3 times. After applying the mydriatic agent, respiratory rate, heart rate, oxygen saturation, and pain score were obtained.

ROP screening was performed by two trained Medical Retina fellows who have sufficient knowledge and experience in identifying ROP location and staging and the sequential retinal changes seen in ROP. Before the actual conduct of the trial, they both examined ten neonates in conventional

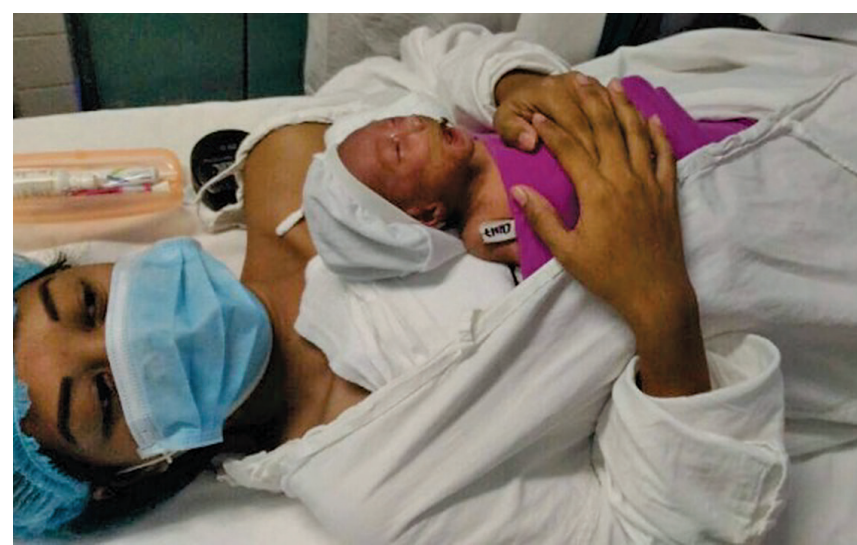

Figure 2. Reverse-KMC.

position and ten neonates in reverse KMCto determine the level of agreement. The two examiners had a Cohen's kappa $>0.80$.

Pre-treating the eyes with a topical anesthetic agent such as proparacaine hydrochloride 0.5\% (1-2 drops, 30-60 seconds during the screening) was applied. The eye speculum was inserted gently by the ophthalmologist 30 seconds after the anesthetic was administered. Both eyes were examined by indirect ophthalmoscope and wire vectis (for scleral indentation), as seen in Figure 3.
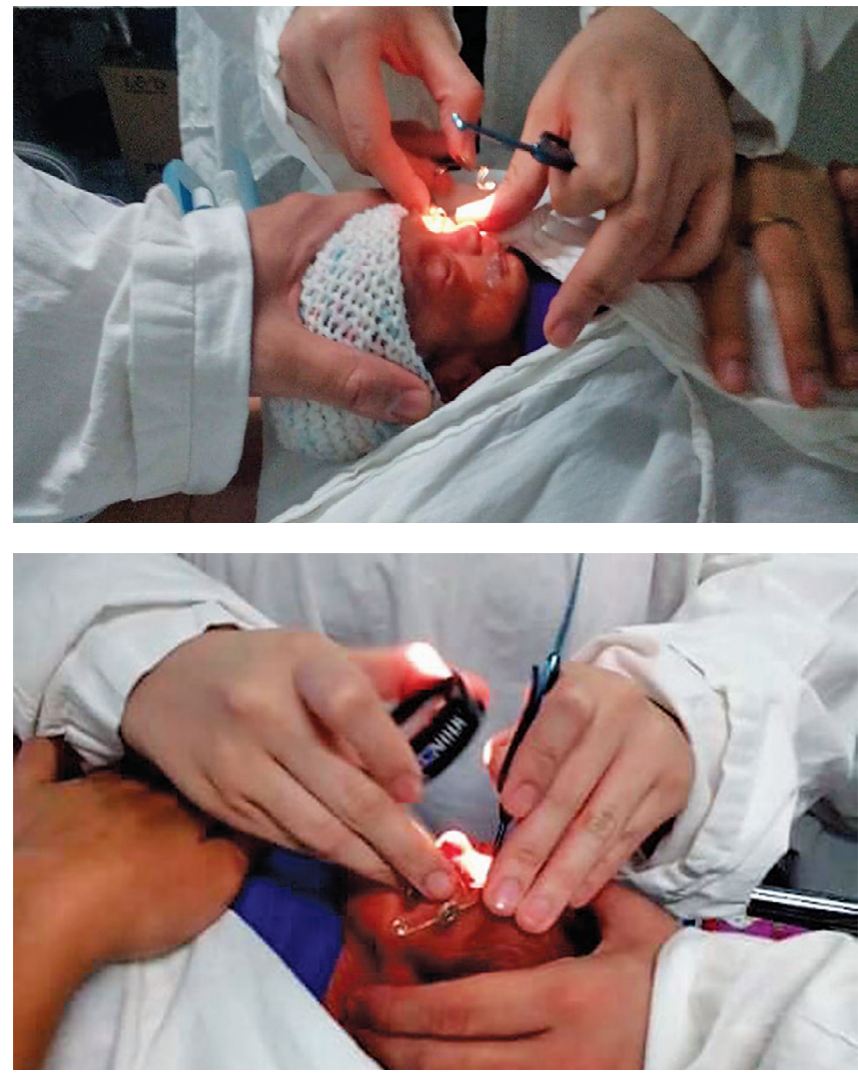

Figure 3. ROP Procedure. 
Baseline physiologic parameters and PIPP scores were done before the examination. These were repeated 30 seconds after removing both speculums, which was done after both eyes were examined. Adverse events due to mydriatic drugs such as apnea were monitored after mydriatic application until the removal of both speculums. Only one trained individual was involved in the measurement of vital parameters and PIPP scoring of the neonate.

The duration of the ROP procedure was recorded. This was from the instillation of an anesthetic agent to the removal of the eye speculum. An operator assessment form was given to the Medical Retina Fellow after the procedure.

\section{Outcome Measures}

\section{Main Outcome}

- The primary outcome in this trial was a reduction in infant pain intensity measured by the Premature Infant Pain Profile (PIPP) and improvement in physiologic parameters such as higher oxygen saturation, lower heart rate but within a normal level, and lower respiratory rate within normal levels.

\section{Secondary Outcome}

- Reduction in adverse effects of mydriatic agents such as apnea.

- Shorter procedural time during ROP screening.

- Operator's satisfaction with the reverse-KMC position during ROP screening.

\section{Data encoding}

Data cleaning and checking were done before data analysis by generating summary statistics and marginal frequencies. All data collected were encoded and stored in Microsoft Excel, and statistical analyses were carried out using Stata 13 MP64.

\section{Statistical Analysis}

Analysis of Covariance (ANCOVA) was used to determine if there was a significant change between the mean values of the physiological parameters between neonates under the conventional position and R-KMC after applying mydriatics and during the ROP screening examination. The initial values of physiological parameters were used as the covariate. The $\mathrm{p}$-values computed considered the necessary adjustment based on the baseline values of the physiological parameters of the neonate.

\section{RESULTS}

\section{Baseline Characteristics}

Baseline characteristics did not statistically differ between the two groups, as seen in Table 1 . The study showed 52 male and 48 female preterm infants. The average birth weight and pediatric age were $1411.72 \pm 301.89$ grams $(\mathrm{p}=0.0706)$ and $30.99 \pm 1.95$ weeks, $(\mathrm{p}=0.2955)$ respectively. During ROP screening, the average post-conceptional age and weight were $34.25 \pm 1.85$ weeks $(\mathrm{p}=0.1861)$ and $1.40 \pm 0.25$ grams $(\mathrm{p}=+0.3316)$ respectively.

\section{Physiologic parameters}

After the application of mydriatics, neonates under the $\mathrm{R}-\mathrm{KMC}$ had a slight increase in mean oxygen saturation $(94.98 \pm 2.67$ vs. $94.88 \pm 3.93 ; p=0.7760)$ and lower mean heart rate $(144.64 \pm 15.06$ vs. $145.34 \pm 11.91 ; \mathrm{p}=0.7719)$. Both were not statistically significant. However, mean respiratory rates were significantly lower among the R-KMC group $(50.26 \pm 6.18$ vs. $51.6 \pm 8.35 ; \mathrm{p}=0.0191)$. $R R$ in the control group is 37 to 84 versus 40 to 68 in the R-KMC group. There was at least $5 \mathrm{~min} / \mathrm{change}$ of the respiratory rate from the baseline, as seen in Table 2.

During ROP screening, neonates under the R-KMC had a slight increase in mean oxygen saturation $(95.42 \pm 2.20$ vs. $94.88 \pm 2.54 ; \mathrm{p}=0.2578)$, decrease in both mean heart rate $(142.3 \pm 10.05$ vs. $144.12 \pm 18.39 ; \mathrm{p}=0.5410)$ and respiratory rate $(49.72 \pm 4.56$ vs. $51.22 \pm 8.73 ; \mathrm{p}=0.2873)$. All did not reach statistical significance, as also seen in Table 2.

\section{Pain intensity assessment}

The R-KMC group had a significantly lower mean PIPP score $(6.47 \pm 2.06$ vs. $8.76 \pm 2.80 ; \mathrm{p}<0.0001)$ after the application of mydriatics compared to the control group. The $\mathrm{R}-\mathrm{KMC}$ group also had significantly lower mean PIPP scores during the ROP screening than the control group (7.58 \pm 2.05 vs. $9.82 \pm 2.90$; p-value: $<0.0001$ ), as seen in Table 3 .

When the PIPP scores were categorized as seen in Table 4 into 'minimal to no pain' (PIPP score < 7), 'moderate pain' (PIPP score $=7$ to 12), or 'severe pain' (PIPP score > 12), more preterm infants experienced 'minimal to no pain after application of mydriatics while on $\mathrm{R}-\mathrm{KMC}$ position than

Table 1. Comparison of baseline characteristics between preterm infants on the R-KMC position and control

\begin{tabular}{lccc} 
& Conventional position ( $\mathbf{n = 5 0 )}$ & $\mathbf{R}-\mathbf{K M C}(\mathbf{n}=\mathbf{5 0})$ & $\mathbf{p}$-value \\
\hline Birthweight (in g) & $1466.94 \pm 340.79$ & $1357.6 \pm 249.94$ & 0.0706 \\
\hline Pediatric age (in gestational age) & $31.18 \pm 2.07$ & $30.77 \pm 1.82$ & 0.2955 \\
\hline Post corrected age during ROP Screening (in weeks) & $34.50 \pm 2.20$ & $34.01 \pm 1.39$ & 0.1861 \\
\hline Weight during ROP Screening (in g) & $1.43 \pm 0.28$ & $1.38 \pm 0.23$ & 0.3316 \\
\hline
\end{tabular}

${ }^{*}$ cell contents are presented as mean $\pm S D$

$p$-value computed using $t$-test for two means 
KMC in Decreasing Adverse Physiological Events and Pain during ROP Screening

Table 2. Comparison of physiological parameters after application of mydriatic agent and during ROP screening between preterm infants on R-KMC and Control

\begin{tabular}{|c|c|c|c|c|c|}
\hline & & Conventional position & R-KMC & Difference (CC - R-KMC) & p-value \\
\hline \multirow{3}{*}{$\begin{array}{l}\text { After application of } \\
\text { mydriatic agents }\end{array}$} & Oxygen saturation & $94.88 \pm 3.93$ & $94.98 \pm 2.67$ & -0.1 & 0.7760 \\
\hline & Heart rate & $145.34 \pm 11.91$ & $144.64 \pm 15.06$ & -0.7 & 0.7719 \\
\hline & Respiratory rate & $51.6 \pm 8.35$ & $50.26 \pm 6.18$ & $1.34^{\wedge}$ & 0.0191 \\
\hline \multirow{3}{*}{$\begin{array}{l}\text { During ROP } \\
\text { Screening } \\
\text { Examination }\end{array}$} & Oxygen saturation & $94.88 \pm 2.54$ & $95.42 \pm 2.20$ & 0.54 & 0.2578 \\
\hline & Heart rate & $144.12 \pm 18.39$ & $142.3 \pm 10.05$ & 1.82 & 0.5410 \\
\hline & Respiratory rate & $51.22 \pm 8.73$ & $49.72 \pm 4.56$ & 1.5 & 0.2873 \\
\hline
\end{tabular}

${ }^{*}$ cell contents are presented as mean $\pm S D$

the $p$-value is adjusted for the baseline physiological parameters, i.e., adjusted for the covariate, which is the baseline value

^ statistically significant at $5 \%$

Table 3. Comparison of PIPP scores after application of mydriatic agent and during ROP screening between preterm infants on R-KMC and Control

\begin{tabular}{lcccc} 
& Conventional position & R-KMC & Difference (CC - R-KMC) & p-value \\
\hline After application of mydriatic agents & $8.76 \pm 2.80$ & $6.47 \pm 2.06$ & $2.29^{\wedge}$ & $<0.0001$ \\
\hline During ROP Screening Examination & $9.82 \pm 2.90$ & $7.58 \pm 2.05$ & $2.24^{\wedge}$ & $<0.0001$ \\
\hline
\end{tabular}

${ }^{*}$ cell contents are presented as mean $\pm S D$

${ }^{\wedge}$ statistically significant at $5 \%$

Table 4. Comparison of categorized PIPP scores after application of mydriatic agent and during ROP screening between preterm infants on R-KMC and Control

\begin{tabular}{|c|c|c|c|c|c|c|}
\hline & \multicolumn{3}{|c|}{ After application of mydriatic agent } & \multicolumn{3}{|c|}{ During ROP Screening } \\
\hline & Conventional position & R-KMC & p-value & Conventional position & R-KMC & p-value \\
\hline \multirow{2}{*}{ Minimal to No Pain } & 11 & 24 & \multirow{2}{*}{0.0064} & 9 & 14 & \multirow{2}{*}{0.2348} \\
\hline & $22 \%$ & $48 \%$ & & $18 \%$ & $28 \%$ & \\
\hline \multirow{2}{*}{ Moderate Pain } & 32 & 25 & \multirow{2}{*}{0.1574} & 31 & 34 & \multirow{2}{*}{0.5294} \\
\hline & $64 \%$ & $50 \%$ & & $62 \%$ & $68 \%$ & \\
\hline \multirow{2}{*}{ Severe Pain } & 7 & 1 & \multirow{2}{*}{0.027} & 10 & 2 & \multirow{2}{*}{0.0138} \\
\hline & $14 \%$ & $2 \%$ & & $20 \%$ & $4 \%$ & \\
\hline
\end{tabular}

conventional position ( $48 \%$ vs. $22 \%$; $\mathrm{p}=0.0064$ ), however, there were significantly fewer infants on R-KMC who experienced severe pain than control ( $2 \%$ vs. $14 \%$; $p=0.027)$ after application of mydriatics, during ROP Screening, more preterm infants experienced minimal to no pain and moderate pain under R-KMC compared with control, but this were both not statistically significant, but there were significantly fewer preterm infants on $\mathrm{R}-\mathrm{KMC}$ who were observed to have severe pain compared with the control ( $4 \%$ vs. $20 \%$; $\mathrm{p}=0.0138$ ) as also seen in Table 4.

\section{Secondary Outcome}

There was no occurrence of apnea after the application of the mydriatic agent. However, one patient in the control group had apnea during the ROP screening procedure.

The procedural time during ROP Screening for R KMC was shorter than the control group $(7.21 \pm 0.51$ vs. $7.39 \pm$ $0.66 ; p=0.8949$ ) but was not statistically significant.

The ophthalmologist's health care provider satisfaction rating revealed that during $\mathrm{R}-\mathrm{KMC}, 8 \%$ had difficulty applying topical anesthetic agent, $16 \%$ had difficulty inserting the eye speculum, and $12 \%$ had difficulty during indirect ophthalmoscopy. In general, $26 \%$ recommended the position during ROP screening, while a majority (70\%) were neutral.

\section{DISCUSSION}

ROP Screening is a painful procedure that will likely result in a stress response. During the screening, procedures such as application of mydriatic agent, insertion of the speculum, sclera depression, manipulation of the globe during visualization, bright light, and physical restraints all contribute to the pain experienced by the preterm infant. The majority (57\%) of our subjects experience moderate pain during the application of mydriatic agent, and $65 \%$ experience moderate pain during the retinal examination.

Based on the PIPP pain assessment tool, moderate pain needs the institution of comfort measures. The current recommendation to reduce pain and discomfort in infants is pre-treatment of the eyes with a topical anesthetic agent 
(proparacaine). Other measures such as comfort care such as nesting, pacifiers, and oral sucrose are recommended. However, despite these recommendations, there are conflicting reports on the efficacy of using a topical anesthetic agent. Saunders et al. have shown no decrease in pain when proparacaine is used during the infant eye examination. ${ }^{15}$ A similar study of Cogen has shown a reduction in pain intensity among those who received the anesthetic, but this is not statistically significant. ${ }^{16}$ Two studies (Mehta and Marsh) showed decreased PIPP scores at speculum insertion and $1^{\text {st }}$ min of examination only. ${ }^{17,18}$ There was no difference at the $5^{\text {th }}$ minute of the procedure in both studies, which indicates that the benefit is not sustained. Other studies by Nesargi and Olsson et al. using dextrose or sucrose as a method of pain relief has not decreased the PIPP pain scores. ${ }^{19,20}$

This study utilizes the Reverse-Kangaroo Mother Care position as an intervention to decrease pain during ROP Screening. Our results show that it reduced stress in terms of pain scores, as documented by the lower PIPP score during the application of mydriatics and ROP screening. The effect of $\mathrm{KMC}$ on pain reduction has been explained as behavioral changes during skin-to-skin contact, which stimulates deep sleep. The pain response is attenuated during deep sleep. It appears to be related to activation of the pain inhibition system via endogenous system modulation. ${ }^{21,22}$ Kangaroo Care may contribute to the activation of the autonomic system, such as reduction in heart rate and respiratory rate that is also seen in our study. However, it did not always reach significant levels.

Our study results were comparable to the study done by Padhi et al. in which changes in heart rate and oxygen saturation were not statistically different between the two groups. However, behavioral changes (discomfort evidenced by eye squeezing, crying and brow bulging) were minimized just after or within 10s of the conclusions of the examination in the majority of the subjects. A similar analgesic effect of $\mathrm{KMC}$ has been observed in studies on BCG vaccination and heel prick in which the PIPP scores were significantly less in the $\mathrm{KMC}$ group than the control group.

Adverse effects directly related to systemic absorption of a mydriatic agent such as apnea have been analyzed in this study. Only one patient developed apnea in this study, and he belonged to the control group. However, the preterm infants have been monitored only during and immediately after the ROP screening. This may lead to missing out on apneic episodes minutes and hours after the procedure.

Regarding time employed during the examination procedure, positioning the infant under R-KMC does not affect the screening duration. Despite some difficulty during the procedure under $\mathrm{R}-\mathrm{KMC}, 26 \%$ still recommended the position during ROP. Still, 70\% were neutral in their preference for this new approach during the ROP screening procedure. There seemed to be more hesitation on the part of the ROP screener early on in the study. On subsequent $\mathrm{ROP}$ screening using the reverse $\mathrm{KMC}$ position, the ROP screener responded more favorably to the R-KMC position and took less time to examine the preterm infant. However, the mother's presence during the painful procedure may be a factor in 'neutral' responses of the ROP screeners.

\section{Limitations of the study}

In our study setting, it was not feasible to rate all the parameters of the PIPP during the retinal exam since facial expressions could not be assessed while the speculum was still in the eye of the preterm infant.

\section{CONCLUSION}

R-KMC significantly reduced pain during ROP screening. It was a low-cost strategy and can be recommended as a position of choice for pain control during ROP screening.

\section{Recommendations}

Further studies are recommended to find the comfort of the ophthalmologist during the ROP screening (height of the table, lighting, pre-counseling of the parents, etc.). Also, dissemination of the study results showing the benefits of R-KMC during ROP screening is essential to decrease the initial hesitancy of the Ophthalmologists to adopt this position.

\section{Statement of Authorship}

All authors participated in the data collection and analysis and approved the final version submitted.

\section{Author Disclosure}

All authors declared no conflicts of interest.

\section{Funding Source}

This study was funded by the Kangaroo Mother Care Foundation Philippines, Inc.

\section{REFERENCES}

1. Craig KD, Whitfield MF, Grunau RV, Linton J, Hadjistavropoulos. Pain in the Preterm Neonate: Behavioral and Physiological Indices. Pain. 1993;52(3):287-99.

2. Anand KJ, International Evidence-Based Group for Neonatal Pain. Consensus Statement for the Prevention and Management of Pain in the Newborn. Arch Pediatr Adolesc Med. 2001; 155(2):173-80.

3. Campbell-Yeo ML, Disher T, Benoit B,Johnston C. Understanding Kangaroo Care and Its Benefits to Preterm Infants. Pediatric Health Med Ther. 2015; 6: 15-32.

4. Bera A, Ghosh J, Singh AK, Hazra A, Tapas S, Munian D. Effect of Kangaroo Mother Care on Vital Physiological Parameters of the Low Birth Weight Newborn. Indian Journal Community Med. 2014; 39(4): 245-9.

5. Dehghani K, Movahed Z, Nasiriani K, Nasiriani K. Kangaroo Mother Care versus Conventional Method on Vital Signs and Arterial Oxygen Saturation Rate in Newborns. Journal of Clinical Neonatology 2015; 4:26-31.

6. Sutar R, Baraha S, Mummidi P. Effects of Kangaroo Mother Care on Common Vital Parameters of Preterm Infants. IJSR. 2015;4(12): 345-7. 
7. Padhi TR, Sareen D, Pradhan L, Jalali S, Sutar S, Das T, et al. Evaluation of Retinopathy of Prematurity Screening in Reverse Kangaroo Mother Care: A Pilot Study. Eye (Lond) 2015; 29:505-8.

8. Chidambaram AG, Manjula S, Adhisivam B, Bhat B. Effect of Kangaroo Mother Care in Reducing Pain Due to Heel Prick among Preterm Neonates: A Crossover Trial. J Matern Fetal Neonatal Med. 2014; 27(5): 488-90.

9. Johnston C, Filion F, Campbell-Yeo M, Goulet C, Bell L, McNaughton K. Kangaroo Mother Care Diminishes Pain from Heel Lance in Very Preterm Neonates: A Crossover Trial. BMC Pediatrics 2008 8:13.

10. Johnston C, Filion F, Campbell-Yeo M, Goulet C, Bell L, McNaughton $\mathrm{K}$, et al. Enhanced Kangaroo Mother Care for Heel Lance in Preterm Neonates: A Crossover Trial. J Perinatol. 2009; 29:51-6.

11. Gao H, Xu G, Dong R, Fu H, Wang D, Zhang H, et al. Effect of Repeated Kangaroo Mother Care on Repeated Procedural Pain in Preterm Infants: A Randomized Controlled Trial. Int J Nurs Stud.2015;52:1157-65.

12. Kotsandy R, Anderson GC, Good M. Skin-to-skin Contact Diminishes Pain from Hepatitis B Vaccine Injection in Healthy Full-term Neonates. Neonatal Netw. 2013; 32(4): 274-80.

13. Hendricks-Muñoz MD, Li Y, Kim YS, Prendergast CC, Mayers R, Louie M. Maternal and Neonatal Nurse Perceived Value of Kangaroo Mother Care and Maternal Care Partnership in the Neonatal Intensive Care Unit. Am J Perinatol. 2013; 30(10): 875-80.

14. Borm GF, Fransen J, Lemmens WA. A Simple Sample Size Formula for Analysis of Covariance in Randomized Clinical Trials. J Clin Epidemiol. 2007; 60:1234-8.
15. Saunders R, Miller K, Hurshell H. Topical Anesthesia During Infant Eye Examination: Does it Reduce Stress? Ann Ophthalmol. 1993; 25436-9.

16. Cogen MS, Parker JS, Sleep TE, Elsas FJ, Metz TH, Mcgwin G. Masked Trial of Topical Anesthesia for Retinopathy of Prematurity Eye Examinations. J AAPOS.2011; 15:45-8.

17. Mehta VA, Mansfield T, Vanderveen DK. Effect of Topical Anesthesia and Age on Pain Scores during Retinopathy of Prematurity of Screening. J Perinat.2010; 30:731-5.

18. Marsh VA, Young WO, Dunaway KK, Kissling G, Carlos R, Jones SM, et al. Efficacy of Topical Anesthetics to Reduce Pain in Premature Infants during Eye Examinations for Retinopathy of Prematurity. Ann Pharmacother. 2005;39:829-33.

19. Nesargi SV, Nithyanandam S, Rao S, Nimbalkar S, Bhat S. Topical Anesthesia or Oral Dextrose for the Relief of Pain in Screening for Retinopathy of Prematurity: A Randomized Controlled Doubleblinded Trial. J Trop Pediatr. 2015 Feb; 61(1): 20-4.

20. Olsson E, Eriksson M. Oral Glucose for Pain Relief during Eye Examination for Retinopathy of Prematurity. J Clin Nurs. 2011; 20:1054-9.

21. Cohen AM, Cook N, Harris MC, Ying GS, Binehaum G. The Pain Response to Mydriatric Eyedrops in Preterm Infants. J Perinatol 2013;33 (6): 462-465.

22. Freire NB, Garcia JB, Lamy ZC. Evaluation of Analgesic Effect of Skin to Skin Contact Compared to Oral Glucose in Preterm Neonates. Pain. 2008;139 (1):28-33.

The Acta Medica Philippina is now accepting original scientific papers, review articles and case reports for its upcoming issues. Please follow the format for submission as indicated in the "Instructions to Authors" elsewhere in the journal. All papers received shall be properly acknowledged. For inquiries and submission of proposals, please email us at actamedicaphilippina.upm@up.edu.ph 